\title{
Regulation of plant innate immunity by three proteins in a complex conserved across the plant and animal kingdoms
}

\author{
Kristoffer Palma, ${ }^{1,2}$ Qingguo Zhao, ${ }^{3}$ Yu Ti Cheng, ${ }^{1,2}$ Dongling Bi, ${ }^{3}$ Jacqueline Monaghan,,4

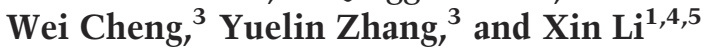 \\ ${ }^{1}$ Michael Smith Laboratories, University of British Columbia, Vancouver, British Columbia V6T 1Z4, Canada; ${ }^{2}$ Genetics \\ Graduate Program, University of British Columbia, Vancouver, British Columbia V6T 1Z3, Canada; ${ }^{3}$ National Institute of \\ Biological Sciences (NIBS), Zhongguancun Life Science Park, Beijing 102206, People's Republic of China; ${ }^{4}$ Department of \\ Botany, University of British Columbia, Vancouver, British Columbia V6T 1Z4, Canada
}

Innate immunity against pathogen infection is an evolutionarily conserved process among multicellular organisms. Arabidopsis SNC1 encodes a Resistance protein that combines attributes of multiple mammalian pattern recognition receptors. Utilizing snc1 as an autoimmune model, we identified a discrete protein complex containing at least three members-MOS4 (Modifier Of snc1, 4), AtCDC5, and PRL1 (Pleiotropic Regulatory Locus 1) - that are all essential for plant innate immunity. AtCDC5 has DNA-binding activity, suggesting that this complex probably regulates defense responses through transcriptional control. Since the complex components along with their interactions are highly conserved from fission yeast to Arabidopsis and human, they may also have a yet-to-be-identified function in mammalian innate immunity.

[Keywords: Innate immunity; Arabidopsis; MOS4; PRL1; AtCDC5; NTC; MAC]

Supplemental material is available at http://www.genesdev.org.

Received April 9, 2007; revised version accepted April 30, 2007.

Innate immunity is the front-line defense system of multicellular eukaryotes and is activated by a limited number of pattern recognition receptors (PRRs). Most PRRs in animals contain leucine-rich repeat (LRR) motifs including Toll-like receptors (TLRs) and proteins with a nucleotide oligomerization domain (NOD) (Akira et al. 2006). There is considerable evidence that plant and animal innate immune systems are conserved as a consequence of convergent evolution (Ausubel 2005). Initiation of induced plant defense signaling involves the recognition of specific pathogen effectors by the products of specialized host RESISTANCE $(R)$ genes (Belkhadir et al. 2004). TIR-NB-LRR-type R proteins combine features of two different classes of PRRs in animal innate immunity: TLRs and NOD proteins (Philpott and Girardin 2004). In the unique Arabidopsis snc1 mutant, a gain-offunction mutation in a TIR-NB-LRR-type $R$ gene results in constitutive defense responses against a wide spectrum of pathogens-the specific pathogen effector detected by wild-type SNC1 in nature is unknown. The snc1 mutation is located in the linker region between the NB and LRR (Zhang et al. 2003). Intriguingly, mutations in NODs are associated with autoimmune disor-

${ }^{5}$ Corresponding author.

E-MAIL xinli@interchange.ubc.ca; FAX (604) 822-2114.

Article is online at http://www.genesdev.org/cgi/doi/10.1101/gad.1559607. ders such as Crohn's disease and Blau syndrome, and mutations in the corresponding linker region of some mammalian NODs result in constitutive self-activation of innate immune responses (Eckmann and Karin 2005).

To identify additional components required for plant innate immunity, a suppressor screen was performed to search for mutations that revert the autoimmune phenotypes of snc1 to wild type. One mutant, modifier of snc1, 4 (mos4), completely abolished enhanced resistance to the virulent pathogens in snc1. MOS4 encodes a nuclear protein homologous to human Breast CancerAmplified Sequence 2 (BCAS2). MOS4 directly interacts in planta with AtCDC5, an atypical R2R3 Myb transcription factor with significant homology with human CDC5L, which is a key component of the spliceosomeassociated PRP Nineteen Complex (NTC). BCAS2 also belongs to NTC. AtCDC5 was previously shown to have sequence-specific DNA-binding activity (Hirayama and Shinozaki 1996); thus it most likely functions as a transcriptional regulator. A third member of the NTC that interacts directly with CDC5L is PLRG1 (Ajuh et al. 2001). In Arabidopsis, one of the PLRG1 homologs, Pleiotropic Regulatory Locus 1 (PRL1), interacted with AtCDC5 in planta. MOS4, AtCDC5, and PRL1 are all required for pathogen resistance in Arabidopsis. Thus, the evolutionarily conserved NTC plays an essential regulatory role in plant innate immunity and may have a similar function in animals. 


\section{Results}

mos4-1 suppresses the autoimmunity-related phenotypes of snc1

In plants, innate immunity against microbial pathogens is governed by $\mathrm{R}$ proteins that are responsible for recognizing pathogen effectors and initiating downstream defense responses (Belkhadir et al. 2004). The most common $\mathrm{R}$ proteins are TIR- and CC-class NB-LRR proteins (Chisholm et al. 2006). Although many $R$ genes have been cloned, the signal transduction events downstream from $\mathrm{R}$ proteins remain scarcely detailed. In order to identify more signaling components downstream from R-protein activation, we carried out a suppressor screen using snc1 (Zhang and Li 2005; Y. Zhang et al. 2005). The mos4-1 snc1 double-mutant plants were the same size as wild type, and had leaves that were less curly than in snc1 (Fig. 1A). Constitutive pBGL2-GUS reporter gene expression in snc1 was completely abolished by mos4-1 (Supplementary Fig. 1A). Furthermore, RT-PCR analysis showed that endogenous $P R-2$ (BGL2) was no longer constitutively expressed in mos4-1 snc1 plants, and that the expression of $P R-1$ was partially suppressed (Supplementary Fig. 1B). Genetic analysis indicated that mos4-1 is a single recessive mutation (data not shown). In addition, defects in MOS4 affected flowering time and male fertility (Supplementary Fig. 2).

The snc1 mutant accumulates high levels of the defense-signaling molecule salicylic acid (SA). Previously, we found that blocking SA synthesis only partially suppressed snc1 phenotypes (Zhang et al. 2003). In mos4-1
A

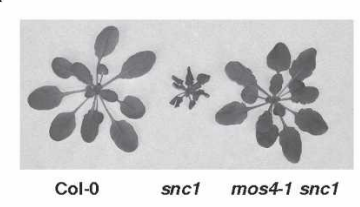

C

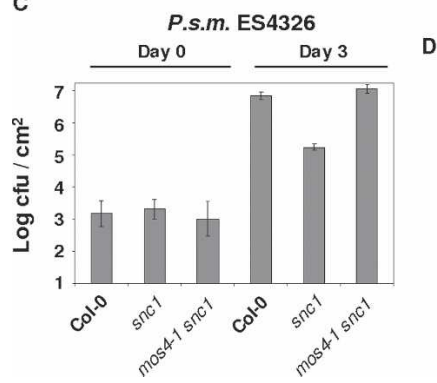

B

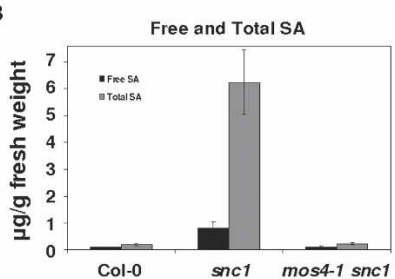

D

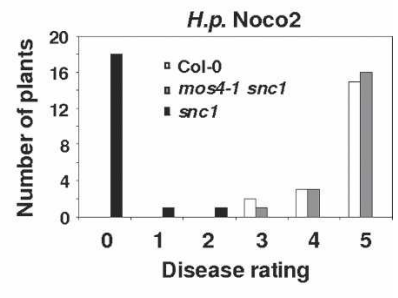

Figure 1. Suppression of snc1-associated morphology, SA accumulation and pathogen resistance by mos4-1. (A) Morphology of wild-type Columbia (Col-0), snc1, and mos4-1 snc1. Image is of representative plants. (B) Free and total SA in leaves of Col-0, snc1, and mos4-1 snc1 plants. (C) Growth of P.s.m. ES4326 at 0 and 3 DPI with $\mathrm{OD}_{600}=0.001$. The log-transformed values presented are averages of four replicates \pm SD. $(D)$ Growth of $H . p$. Noco2 (see the Supplemental Material). Data are presented for 20 plants of each genotype. The experiments were repeated at least twice with similar results. snc1, levels of free SA were about eightfold lower than in snc1, and levels of total SA were $\sim 30$-fold lower than in snc1 (Fig. 1B); these SA levels were comparable with wild-type controls, suggesting that MOS4 functions upstream of SA synthesis.

Two of the most amenable plant-pathogen model systems for the genetic dissection of host responses are those between Arabidopsis and (1) Pseudomonas syringae pv maculicola (P.s.m.) and pv tomato (P.s.t.), two strains of a hemi-biotrophic bacterial species that cause disease symptoms ranging from leaf spots to stem cankers, on a broad range of plant hosts; and (2) Hyaloperonospora parasitica (H.p.), an obligate biotrophic oomycete. Oomycetes grow funguslike hyphae but have cellulosic cell walls and are phylogenetically unrelated to fungi. snc1 is resistant to P.s.m. ES4326 and H.p. Noco2, both of which are normally virulent in Arabidopsis Col-0 plants. While snc1 had no disease symptoms and supported $\sim 50$ times less bacterial growth than wild type at $3 \mathrm{~d}$ post-infection (DPI), mos4-1 snc1 harbored an approximately wild-type titer of bacterial growth (Fig. 1C) and exhibited some disease symptoms (data not shown). Furthermore, mos4-1 completely suppressed resistance to H.p. Noco2 in snc1, as shown in Figure 1D.

\section{MOS4 encodes the Arabidopsis homolog of human BCAS2}

MOS4 (At3g18165) was cloned using a map-based approach (see the Supplemental Material; Supplementary Fig. 3). The mutation in mos4-1 snc1 was found to be an insertion in At3g18165, and 35S-At3g18165 was able to complement the mos4-related phenotypes (Supplementary Fig. 3E,F). In addition, other T-DNA alleles of At3g18165 were able to suppress snc1 (Supplementary Fig, 3G), confirming that MOS4 is indeed At3g18165. Sequence analysis revealed that MOS4 is similar to human BCAS2, with $29 \%$ identity and $48 \%$ similarity at the protein level. An amino acid alignment between Arabidopsis MOS4 and related proteins from other eukaryotes shows highly conserved regions (Supplementary Fig. 4). Since the identity of MOS4 did not reveal the detailed biochemical mechanism of its regulation of innate immunity, we employed three approaches to further elucidate MOS4's detailed modes of action: (1) genetic analysis to identify the pathway in which MOS4 acts, (2) subcellular localization of MOS4, and (3) identification of MOS4-interacting proteins.

MOS4 is essential for signaling in the NPR1-independent pathway

To understand how MOS4 contributes to plant innate immunity, we used a genetic approach to examine the pathway in which it functions. Signaling downstream from snc1 diverges into multiple pathways dependent on different signaling intermediates (Zhang et al. 2003). NPR1, an ankyrin-repeat protein with redox-regulated nuclear translocation, controls basal resistance down- 
stream from SA and regulates $P R-1$ expression through interaction with the TGA subfamily of bZIP transcription factors (Dong 2004). Since npr1-1 does not block PR-2 expression or resistance to virulent P.s.m. ES4326 in the snc1 npr1-1 double mutant, snc1 activates NPR1dependent and independent responses (Zhang et al. 2003). Because mos4-1 completely suppressed the constitutive PR-2 expression of snc1 (Supplementary Fig. 1B), we surmised that MOS4 might be required for the NPR1-independent pathway. To test the epistatic relationship between MOS4 and NPR1, mos4-1 npr1-1 double and mos4-1 npr1-1 snc1 triple mutants were created. When challenged with virulent and avirulent pathogens, mos4-1 completely suppressed the enhanced resistance and morphology of snc1 npr1 in the mos4-1 npr1-1 snc1 triple mutant, and supported at least a 10fold higher titer of bacteria than mos4-1 snc1, suggesting that a defect in MOS4 blocks NPR1-independent pathways downstream from snc1 (Fig. 2A). Furthermore, in the absence of the snc1 mutation, mos4-1 npr1 plants were more susceptible to a normally subclinical concentration of P.s.m. ES4326 than npr1-1 or mos4-1 alone, supporting three- to sevenfold more bacteria 3 DPI (Fig. 2B). The additive nature of the enhanced disease susceptibility (EDS) of mos4-1 and npr1-1 shown by these data suggests that two separate pathways contributing to basal defense are compromised in the double mutant. In addition, mos4-1 npr1-1 was more susceptible to aviru-

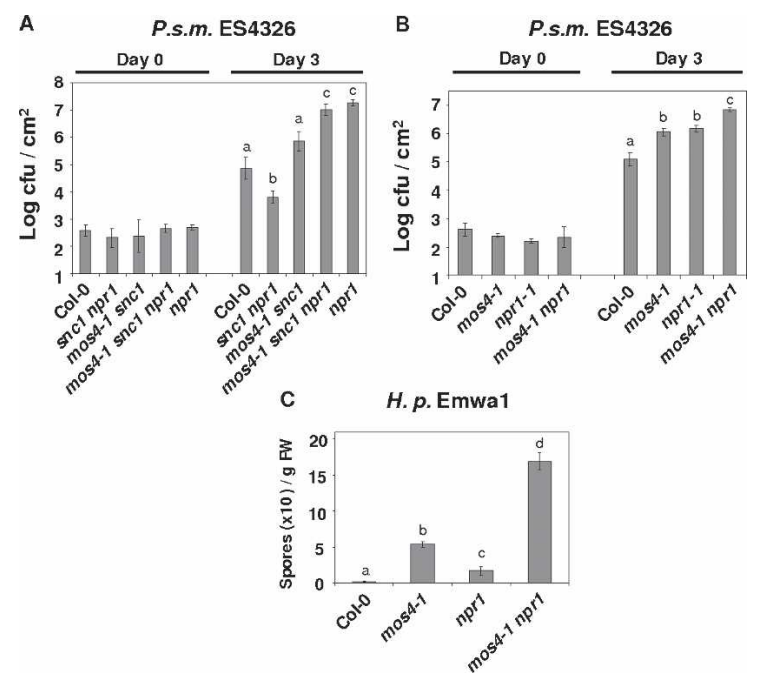

Figure 2. mos4-1 affects the NPR1-independent signaling pathway. (A) Growth of P.s.m. ES4326 in Col-0, snc1 npr1-1, mos4-1 snc1, mos4-1 snc1 npr1-1, and npr1-1 after infiltration with $\mathrm{OD}_{600}=0.0001$. (B) Growth of P.s.m. ES4326 in Col-0, mos4-1, npr1-1, and mos4-1 npr1-1 after infiltration with $\mathrm{OD}_{600}=0.00005$. The log-transformed values presented are averages of four replicates \pm SD. (C) Growth of avirulent H.p. Emwa1 on Col-0, mos4-1, npr1-1, and mos4-1 npr1-1 plants. Results are averages of six replicates \pm SD. The log-transformed values presented are averages of four replicates \pm SD. The experiments were repeated at least twice with similar results. (ad) Statistically significant $(P<0.0001)$ difference as determined by unpaired Student's $t$-test. lent H.p. Emwa1 than either the npr1-1 or mos4-1 single mutants (Fig. 2C), indicating that NPR1-independent signaling downstream from $\mathrm{R}$ protein activation-in this case, RPP4-is mediated by MOS4.

To date, a fairly exclusive group of NPR1-independent defense signaling components has been described; these include MOS2, a putative RNA-binding protein isolated earlier from the snc1 suppressor screen (Y. Zhang et al. 2005), and FMO1 (FLAVIN-DEPENDENT MONOXYGENASE 1), a positive regulator of EDS1 signaling that is independent of SA accumulation (Bartsch et al. 2006). Although the genetic relationship of FMO with NPR1 remains to be determined, it is likely to be NPR1 independent.

Because the mos4-1 single mutant is able to accumulate SA in response to avirulent pathogen infiltration (Supplementary Fig. 5), MOS4 does not seem to contribute to SA accumulation caused by pathogen infection. The reason why mos4-1 snc1 has low SA is probably due to the SA-independent pathway having a positive amplification role in SA synthesis (Y. Zhang et al. 2005). A schematic model of MOS4 signaling in plant innate immunity is shown in Supplementary Figure 6.

\section{MOS4 is a nuclear protein}

To determine the subcellular localization of MOS4, GFP was cloned in-frame at the C terminus of MOS4 and transformed into mos4-1 plants. GFP fluorescence was detected exclusively in the nucleus of various cell types in several independent MOS4-GFP transgenic lines, including guard cells (Fig. 3A), epidermal cells, and root cells (data not shown), suggesting that MOS4 is a nuclear protein. When MOS4-GFP was transformed into mos4-1 snc1 plants and the transgenic progeny examined, all of the nine transgenic plants selected exhibited snc1 phenotypes, indicating that the expression of MOS4-GFP driven by its native promoter complements the mos4-1 mutation in the snc1 background (Fig. 3B). In addition, MOS4-GFP complemented the EDS phenotype of the mos4-1 single mutant (Fig. 3C). Although mos4-1 with MOS4-GFP is wild-type-like, whereas mos4-1 snc1 with MOS4-GFP is snc1-like, we did not observe any difference in GFP localization or intensity of GFP fluorescence between the two genetic backgrounds (data not shown).

MOS4 interacts in yeast with AtCDC5, an atypical R2R3 Myb transcription factor homologous to human CDC5L

Analysis of the MOS4 amino acid sequence predicted that the C-terminal half had the potential to form an amphipathic $\alpha$-helix, a motif known to mediate proteinprotein interactions through parallel two-stranded coiled-coil structure. We thus employed a GAL4-based yeast two-hybrid screen to search for proteins that are capable of interacting with MOS4. One of the interacting proteins found in the screen was AtCDC5 (Supplemen- 
A

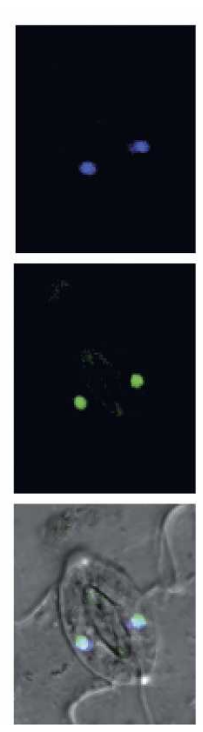

B

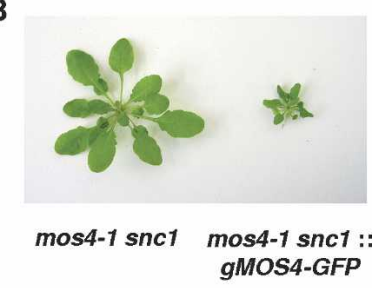

C

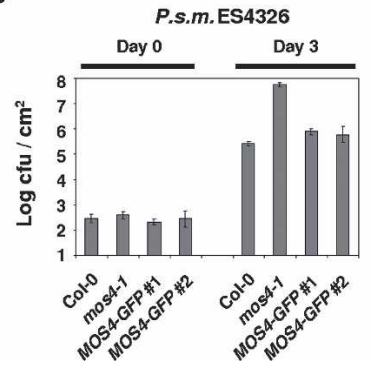

Figure 3. Subcellular localization of MOS4. (A) Guard cells of transgenic mos4-1 plants expressing the genomic MOS4:GFP fusion protein were examined by confocal microscopy; a representative plant is shown. (Top panel) DAPI staining of the nucleus. (Middle panel) MOS4-GFP fluorescence. (Bottom panel) Merged fluorescence channels and bright-field image. $(B)$ Morphology of mos4-1 snc1 complemented with gMOS4-GFP. (C) Growth of P.s.m. ES4326 in Col-0, mos4-1, and two independent complementing lines of gMOS4-GFP transformed into mos4-1 mutants at 0 and 3 DPI, showing complementation of EDS in mos4-1 gMOS4-GFP. A subclinical concentration of P.s.m. $\left(\mathrm{OD}_{600}=0.0001\right)$ was infiltrated. The log-transformed values presented are averages of four replicates \pm SD. The experiment was repeated twice with similar results.

tary Fig. 7A), an 844-amino-acid polypeptide with similarity to R2R3-type MYB transcription factors that exhibits in vitro sequence-specific DNA-binding activity (Hirayama and Shinozaki 1996). To confirm in planta interaction of MOS4 and AtCDC5, HA or GFP was cloned in-frame at the $\mathrm{C}$ ternimus of MOS4 driven by its own promoter and transformed into mos4-1. The fusion clone is able to fully complement mos4-related phenotypes (data not shown), indicating that the fusion proteins function the same as the wild type. Immunoprecipitation (IP) using Anti-GFP Microbeads followed by Western blot analysis with AtCDC5 antibody showed that AtCDC5 coimmunoprecipitated with MOS4-GFP (Fig. 4A). Coimmunoprecipitation (co-IP) with Anti-HA Microbeads yielded similar results (data not shown).

The human homologs of MOS4 and AtCDC5, BCAS2 (also known as hSPF27) and hCDC5L, respectively, have been isolated as components of the multiprotein spliceosome complex by proteomic analysis (Neubauer et al. 1998; Zhou et al. 2002). Using different purification strategies and mass spectrometry, hCDC5L copurified with at least five non-small nuclear RNA (snRNA) proteins in a discrete complex, one of which was BCAS2, the MOS4 homolog (Ajuh et al. 2000). CDC5L orthologs in Schizosaccharomyces pombe and Saccharomyces cerevisiae, Cdc5p and Ceflp, respectively, also copurified

with a core complex nearly identical in composition to that in human cells (Ohi and Gould 2002). The complex containing Ceflp was termed the PRP NTC, based on the identity of another complex member, Prp19p (Cwf8p in S. pombe) (Tsai et al. 1999). The hNTC has also been termed the NMP200 Complex and PSO4 Complex (Ohi and Gould 2002). In vitro immunodepletion of the orthologous human NTC from HeLa nuclear extracts inhibits formation of pre-mRNA splicing products (Ajuh et al. 2000). Taken together, the conserved interaction between MOS4 and AtCDC5 homologs in yeast, Arabidopsis, and human suggests an evolutionarily conserved function of MOS4 through its interaction with AtCDC5.

\section{AtCDC5 interacts with PRL1}

A third component of the human NTC is PLRG1, a WD40 repeat protein shown to bind directly to CDC5L (Ajuh et al. 2001). An Arabidopsis homolog of PLRG1, the previously described PRL1(At4g15900), encodes a conserved nuclear WD protein that functions as a pleiotropic regulator of glucose and hormone responses (Nemeth et al. 1998). Using a yeast two-hybrid assay, we showed that AtCDC5 interacted directly with PRL1 (Supplementary Fig. 7B). In addition, PRL1-GFP was localized to the nucleus (Supplementary Fig. 8). To test for in planta interaction, GFP or HA tag was cloned in-frame at the C terminus of PRL1 and transformed into prl1-1. The fusion clone fully complemented all the prl1 phe-
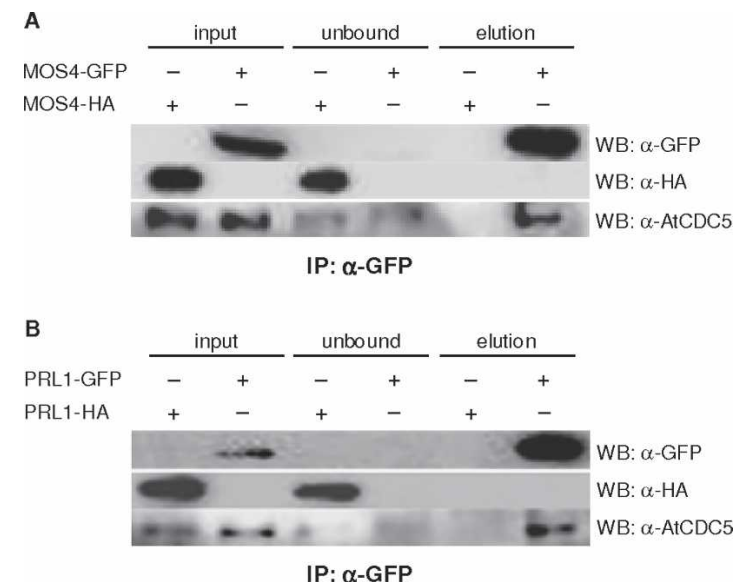

Figure 4. MOS4, AtCDC5, and PRL1 interact in planta. (A) AtCDC5 coimmunoprecipitates with MOS4-GFP in nuclear extracts from mos4-1 complemented by gMOS4-GFP transgenic plants. (B) AtCDC5 coimmunoprecipitates with PRL1-GFP in nuclear extracts from prl1-1 complemented by $g P R L 1-G F P$ transgenic plants. The aerial plant tissue was harvested for nuclear fractionation, and nuclear extracts were subjected to IP as indicated. Equal amounts by number of nuclei in nuclear extracts (input) and flow-through supernatant after IP (unbound) were loaded for immunoblotting. The elution fraction for immunoblotting with anti-GFP or anti-HA antibody was $\sim 20$ times more concentrated than the nuclear extract, and that of anti-AtCDC5 antibody was $\sim 40$ times more concentrated. Protein from MOS4-HA $(A)$ and PRL1-HA $(B)$ transgenic plants were used as negative controls in anti-GFP IPs. 
notypes (data not shown). Using an IP-Western procedure similar to what was done with MOS4, it was demonstrated that AtCDC5 coimmunoprecipitated with PRL1-GFP (Fig. 4B). Co-IP with HA column yielded similar results (data not shown).

\section{A mutation in AtCDC5 suppresses snc1}

To determine if AtCDC5 is also involved in snc1-dependent signaling, a T-DNA insertion line (SAIL_207_F03) in the $5^{\prime}$-untranslated region (UTR) of AtCDC5 (At1g09770) was obtained from the Arabidopsis Biological Resource Center (ABRC). The morphological phenotypes of Atcdc5-1 were very similar to mos4-1; like mos4-1, Atcdc5-1 flowered late, but was slightly smaller than mos4-1 and exhibited complete male sterility (Supplementary Fig. 2). All of these phenotypes were complemented by a genomic clone of wild-type AtCDC5 (data not shown). The mutation in AtCDC5 partially suppressed snc1 morphology, as the Atcdc5-1 snc1 double-mutant plants grew bigger and had leaves that were less curly than in snc1 (Fig. 5A). Atcdc5-1 snc1 plants also partially suppressed $P R$ gene expression (Fig. $5 \mathrm{~B})$ and supported higher bacterial growth than snc1 (Fig. 5C). Thus, the mutation in AtCDC5 suppressed en-

A
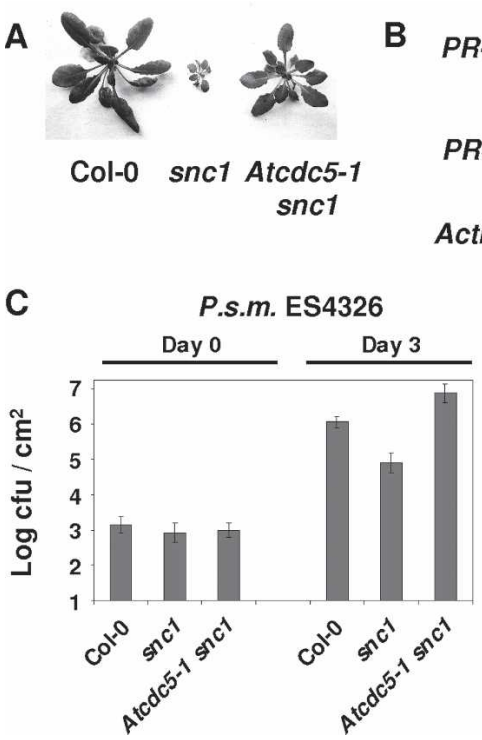

Figure 5. Suppression of snc1 by Atcdc5-1. (A) Phenotypes of Col-0, snc1, and Atcdc5-1 snc1 plants showing suppression of snc1 morphology by Atcdc5-1. Image is of representative plants. (B) PR-2 (BGL2) and $P R-1$ gene expression in Col-0, snc1, and Atcdc5-1 snc1. RNA was extracted from 3-wk-old plants grown on MS medium and reverse-transcribed to obtain total cDNA. The cDNA samples were normalized by real-time PCR using an Actin 1 probe. $P R-1, P R-2$, and Actin 1 were amplified by 27 cycles of PCR using equal amounts of total cDNA. PCR products were analyzed by agarose gel electrophoresis and ethidium bromide staining. The results reported are representative of several experiments. (C) Atcdc5-1 suppresses enhanced resistance of snc1 to virulent P.s.m. ES4326, after infiltration at $\mathrm{OD}_{600}=0.001$. The experiment was repeated twice with similar results. hanced disease resistance responses in snc1 similar to mutations in MOS4. Subsequently, a second T-DNA insertion line (GABI_278B09) in an exon of AtCDC5 (Atcdc5-2) was obtained and determined to be allelic to Atcdc5-1. Atcdc5-2 also suppressed snc1 (data not shown).

\section{MOS4, AtCDC5, and PRL1 are all required for basal defense}

Three T-DNA insertions in PRL1 were obtained from the ABRC. The morphological phenotypes of all three prl1 alleles were identical to each other and similar to those of mos4-1 and Atcdc5-1, except with no flowering time defect (Supplementary Fig. 2). In addition, a genomic clone of wild-type $P R L 1$ was able to fully complement prl1 phenotypes in 20 out of 22 transgenic $\mathrm{T} 1$ plants (data not shown). Because PRL1 and SNC1 are very closely linked on chromosome 4 , we were unsuccessful in identifying the prl1 snc1 double mutant (data not shown).

Since homologs of MOS4, AtCDC5, and PRL1 all belong to the NTC in fission yeast and human, an immediate question is whether or not these three genes affect innate immunity in a similar manner. We tested the mos4, Atcdc5, and prl1 single mutants for EDS to a subclinical concentration of the virulent bacterial pathogen P.s.m. ES4326. At 3 DPI, disease symptoms were evident in infected leaves of all three mutants and absent in the wild-type Col-0 control (data not shown). Furthermore, mos4 alleles and Atcdc5-1 consistently supported $\sim 25-$ to 50 -fold more bacterial growth than wild-type plants, while prl1 alleles supported an even higher titer of bacteria (at least 10-fold further) in the infected leaves, indicating that MOS4, AtCDC5, and PRL1 are all required for basal resistance to P.s.m. ES4326 (Fig. 6A). Similarly, mos4, Atcdc5, and prl mutants exhibited enhanced susceptibility to other virulent pathogens such as H.p. Noco2 (data not shown) and Pseudomonas syringae pv tomato (P.s.t.) DC3000 (Supplementary Fig. 9A).

Since alleles of prl1 are consistently more susceptible than alleles of mos4 or Atcdc5 (Fig. 6A), we crossed mos4-1 with Atcdc5-1 or prl1-1 to screen for respective double mutants to see if each putative complex member contributes quantitatively to plant immunity. We were unable to identify a mos4-1 Atcdc5-1 or mos4-1 prl1-1 double-mutant plant in the $\mathrm{F}_{2}$ generation. After selecting several $\mathrm{F}_{2}$ plants homozygous for one mutation and heterozygous for the other by PCR, for both mutant combinations, we were unable to find double-homozygous plants in several $F_{3}$ populations of $>100$ plants. This strongly suggests that mos4-1 Atcdc5-1 and mos4-1 pr11-1 double-homozygous plants are probably lethal.

To assay whether these mutants affected $P R$ gene expression during pathogen infection, mos4-1, Atcdc5-1, prl1-1 and npr1-1 plants were inoculated with a clinical dose of virulent P.s.m. ES4326 and the RNA was extracted after $24 \mathrm{~h}$. Real-time RT-PCR revealed that $P R-1$ gene expression was absent in npr1-1 and much lower in the other mutants tested, compared with wild type (Supplementary Fig. 9B). 
A

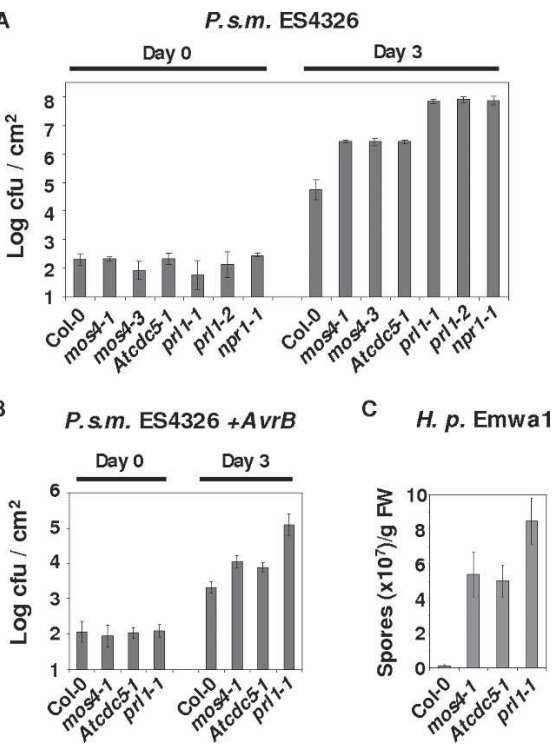

Figure 6. Characterization of mos4, Atcdc5, and prl1 in innate immunity. (A) Growth of P.s.m. ES4326 in Col-0, mos4-1, mos4-3, Atcdc5-1, prl1-1, prl1-2, and npr1-1 single mutants after infiltration at $\mathrm{OD}_{600}=0.0001 .(B)$ Growth of avirulent P.s.m. ES4326-expressing AvrB. The log-transformed values presented are averages of four replicates \pm SD. $(C)$ Growth of avirulent $H . p$. Emwal on Col-0, mos4-1, Atcdc5-1, and prl1-1 plants. Results are averages of six replicates \pm SD. The experiments were repeated three times with similar results.

Is enhanced susceptibility to P.S.M ES4326 in these mutants caused by a deficiency in SA accumulation? To answer this question, we inoculated quarter leaves of Col-0, mos4-1, Atcdc5-1, and prl1-1 with a high dose of avirulent P.s.m. ES426 carrying $A v r B$ and collected the remaining leaf tissue $24 \mathrm{~h}$ later, at which time macroscopic cell death was observed. Total SA was extracted and measured in pathogen- versus mock-inoculated leaves. As seen in Supplementary Figure 5, SA was able to accumulate in all three mutants as in wild type in response to an avirulent pathogen, indicating that MOS4 and its interacting partners do not contribute to SA accumulation after infection.

To test if defects in MOS4, AtCDC5, or PRL1 affected nonhost resistance, we inoculated plants with a high dose of P.s.t. DC3000 lacking $\operatorname{HrpA}$, which encodes a pilus subunit required for the secretion of effectors into the host cytoplasm, and assayed for bacterial growth (Supplementary Fig. 10A). At 4 DPI, there was insignificant growth of P.s.t. DC3000 hrp $A^{-}$in wild-type and all three mutant plants, suggesting that no defect in nonhost resistance exists as in mutants such as nho1 (Lu et al. 2001). We also tested the response of these mutants to a model pathogen-associated molecular pattern (PAMP), flg22, the peptide epitope of the bacterial flagellin protein that is recognized by the receptor-like kinase FLS2 (Felix et al. 1999; Gomez-Gomez et al. 1999). The defense responses mediated by FLS2 are associated with a measurable inhibition of seedling growth upon prolonged treatment with flg22 (Gomez-Gomez et al. 1999).
Ten days after application of $20 \mu \mathrm{M}$ flg22, seedling growth inhibition was similar in wild-type Col-0 and in mos4-1, Atcdc5-1, and prl1-1 seedlings compared with growth in media alone (Supplementary Fig. 10B,C). In addition, injection of flg22 peptide followed by inoculation with P.s.t. DC3000 24 h later resulted in enhanced resistance and consequently lower bacterial growth at 2 DPI versus mock treatment in wild type and in all three mutants (Supplementary Fig. 10D). The Arabidopsis ecotype Wassilewskija-0 (WS-0), which has a natural defect in FLS2, and the fls2 mutant in Col-0 were not responsive to flg22 in either experiment, as expected. These data suggest that response to flg 22 is not compromised in mos4-1, Atcdc5-1, or pr11-1, although we cannot exclude the possibility that these mutants are compromised in an FLS2-independent response to other PAMPs.

\section{MOS4, AtCDC5, and PRL1 are all essential for $R$-protein-mediated resistance}

To test if the mos4, Atcdc5, and prl1 mutations affect resistance mediated by $\mathrm{R}$ proteins other than $\mathrm{SNC1}$, we tested several $A v r-R$ combinations that result in incompatible interactions in Arabidopsis. At least two distinct disease resistance pathways have been described for Arabidopsis, governed by R-protein structural type rather than pathogen class (Aarts et al. 1998). The $R$ genes RPM1 (CC-NB-LRR type) and RPP4 (TIR-NB-LRR type) confer resistance to bacterial and oomycete pathogens expressing specific cognate avirulence gene products (Grant et al. 1995; van der Biezen et al. 2002). mos4-1, Atcdc5-1, and pr11-1 were more susceptible than wild type to both avirulent P.s.m. ES4326 carrying AvrB (RPM1-dependent resistance) and avirulent H.p. Emwal (RPP4-dependent resistance), based on higher growth of pathogens in both mutants (Fig. 6B,C). Unlike EDS1 and NDR1, which are specifically required for signaling of TIR-NB-LRR and CC-NB-LRR-type R proteins, respectively, MOS4, AtCDC5, and PRL1 are required for resistance mediated by both types of $\mathrm{R}$ proteins. Other proteins required for both TIR and CC-NB-LRR R proteins include SGT1b, RAR1, and HSP90, which seem to function in stabilization of the R-protein complex rather than in downstream signaling (Austin et al. 2002; Azevedo et al. 2002; Hubert et al. 2003).

Because mutants in MOS4, AtCDC5, and PRL1 exhibit very similar phenotypes and defects in basal and specific defense responses, MOS4 and PRL1 directly interact with AtCDC5, and homologs of all three proteins belong to the NTC in humans and yeast, we speculate that these three proteins must function together in a multiprotein complex orthologous to the human NTC to regulate innate immunity in Arabidopsis. We term this multiprotein assembly the MOS4-Associated Complex (MAC).

\section{Discussion}

The Arabidopsis MAC and RNA splicing

It is reasonable to propose that the Arabidopsis MAC is orthologous to the NTC, which has been indicated to be 
essential for splicing (Ajuh et al. 2001), based on studies in human and yeast. Since the majority of plant genes are interrupted by noncoding introns, a defect in general splicing of mRNA would likely result in catastrophic phenotypes or death. For example, several general splicing mutants are embryo lethal, based on data in the SeedGenes database (Tzafrir et al. 2003). Since mutations in MOS4, PRL1, or AtCDC5 alone only have minor effects on the plants' normal growth and development, it is unlikely that the protein products of these genes are essential components of splicing. However, since mos4-1 Atcdc5-1 and mos4-1 prl1-1 double-homozygous mutants seem to be lethal, MAC as a whole could be required for an essential process, such as spliceosome assembly, as suggested from studies in yeast and humans (Tarn et al. 1993; Grillari et al. 2005)

In some instances, the splicing machinery can process the same pre-mRNA differently by selectively joining different exons or retaining specific introns. This alternative splicing potentially leads to the generation of structurally and/or functionally distinct proteins. Several TIR-NB-LRR-class $R$ genes, along with TLRs in animals, are alternatively spliced, although the functional relevance is unknown (Jordan et al. 2002). For example, the stoichiometry of two tobacco $\mathrm{N}$-gene-derived splice variants undergoes a 50 -fold change in their relative molar ratios upon pathogen challenge (Dinesh-Kumar and Baker 2000). The Arabidopsis R gene RPS4 produces alternative transcripts with truncated ORFs. The dominant alternative RPS4 transcripts are generated by retention of intron 3 or introns 2 and 3 , which contain inframe stop codons and lie downstream from the NBencoding exon-the combined presence of regular and alternative RPS4 transcripts is necessary for RPS4 function in resistance (Zhang and Gassmann 2003).

Although the functional relevance of alternative splicing in innate immunity is unknown, the association of the NTC with the spliceosome led us to hypothesize that MOS4, AtCDC5, and PRL1 may be involved in alternative splicing. We tested several alternatively spliced genes, including RPS4, AtSRp30, AtSRp34, U1snRNP, $A N P 1$, and POT1, for relative levels of transcript variants of each in the mos4-1, Atcdc5-1, and prl1-1 mutants by real-time RT-PCR. In all cases, there were no significant differences in transcript variant levels between wild type and the mos4-1, Atcdc5, and prl1 mutants (Fig. 7). Our data suggest that MOS4, AtCDC5, and PRL1, while possibly associated with a spliceosome complex, are individually not involved in general or alternative RNA splicing. We cannot discount the possibility that the intact MAC complex is required for spliceosome assembly as the NTC is in yeast, or that the individual members regulate the splicing of an unknown RNA species.

\section{What is the function of the MAC and NTC in plants and animals?}

In human cells, in addition to being associated physically with the spliceosome, the MOS4 ortholog BCAS2 and hCDC5L have been reported to have roles in cancer

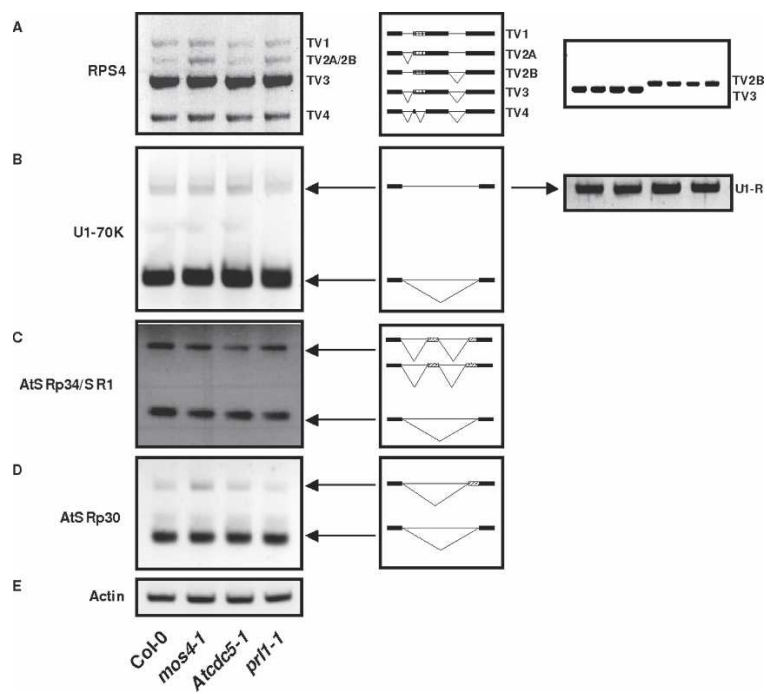

Figure 7. Levels of transcript variants in several alternatively spliced genes in Col-0, mos4-1, Atcdc5-1, and prl1-1. RNA was extracted from leaves and reverse-transcribed to obtain total cDNA. The cDNA samples were normalized by real-time PCR using an Actin 1 probe. PCR products were analyzed by agarose gel electrophoresis and ethidium bromide staining. Schematic diagrams of alternative splicing are shown at right. (A) Alternative splicing of RPS4 results in five transcript variants (TV) of different sizes, as determined by PCR using nested primers as previously described (Zhang and Gassmann 2003). In this case, TV1 retained both introns in the schematic diagram, whereas TV2 was composed of two transcript species of similar size lacking either the first intron (TV2A) or second intron (TV2B). TV3 corresponds to the regular transcript with both introns removed. An additional cryptic intron was spliced out in TV4. Primers were designed across intron/exon boundaries to specifically amplify TV3 and TV2B from diluted PCR products of the distal nested primers. As determined by real-time PCR and shown in the far right panel, there was no significant difference in relative abundance of either alternatively spliced RPS4 transcripts between Col-0, mos4-1, Atcdc5-1, and pr11-1. (B) Alternative splicing of $U 1-70 \mathrm{~K}$ featuring intron retention (SavaldiGoldstein et al. 2003). Primers were designed across intron/exon boundaries to specifically amplify the retained intron transcript of $U 1-70 \mathrm{~K}$, and relative abundance in Col-0 and MAC mutants was assayed by real-time PCR; as determined by real-time PCR and shown in the far right panel, there was no significant difference in relative abundance of retained intron $U 1-70 \mathrm{~K}$ transcript (U1-R). (C) AtSRp34/SR1 showing 5' and 3' alternative splicing (Savaldi-Goldstein et al. 2003). (D) AtSRp30 showing 5' and $3^{\prime}$ alternative splicing (Savaldi-Goldstein et al. 2003). (E) Actin control, 30 cycles. Alternative splicing of ANP1 and POT1 (Tani and Murata 2005) in the MAC mutants resulted in similar patterns (data not shown).

malignancy, apoptosis, estrogen receptor-mediated transcription, and DNA repair (Lee et al. 2002; Maass et al. 2002; Mahajan and Mitchell 2003; Qi et al. 2005; N. Zhang et al. 2005). The detailed biochemical and biological function of BCAS2, hCDC5L, and other members of the human NTC remains elusive in part because of the challenge of genetic analysis at a whole-organism level. Our analysis of the mos4, Atcdc5, and prl1 mutants highlights the advantage of using Arabidopsis genetics 
to dissect biological processes unambiguously and provides an exceptional opportunity to define the function of the NTC. The NTC is an evolutionarily conserved protein complex that interacts in an as-yet-undetermined way with the spliceosome. Future yeast two-hybrid or proteomic efforts using MOS4 epitope-tagged lines will enable us to identify more Arabidopsis components of the complex and analyze their functions using reverse genetic approaches. It is important to note that, although we show that MOS4, AtCDC5, and PRL1 are required for innate immunity, there is as of yet no direct evidence that their interactions are required for innate immunity.

Defects of MOS4, PRL1, and AtCDC5 are confined to innate immunity, flowering time, and fertility, indicating that targets of MAC/AtNTC regulation are limited. Members of the human NTC include PSO4, an E3 ubiquitin ligase, hCDC5L, a transcription factor, and several proteins with protein interaction domains (e.g., BCAS2 and PLRG1), hinting at a complex regulatory module (Loscher et al. 2005; N. Zhang et al. 2005). Since AtCDC5 has sequence-specific DNA-binding capability (Hirayama and Shinozaki 1996), the simplest and most likely explanation is that the MAC fulfills its role through regulating transcription of downstream target genes. The spliceosome may assist the NTC in fulfilling this regulatory function. Alternatively, the NTC may merely colocalize with the spliceosome based on its association with the nuclear structure. A third possibility could be that the MAC contributes to regulatory processes mediated by microRNA (miRNA) and small interfering RNA (siRNA), both of which have been shown recently to be involved in the regulation of development and innate immunity (Katiyar-Agarwal et al. 2006; Navarro et al. 2006). It is interesting that endogenous small RNAs are also involved in the regulation of developmental processes, such as flowering time via FLC (Swiezewski et al. 2007). Future detailed genetic and biochemical analysis using Arabidopsis mac mutants will help reveal these underlying mechanisms. It will also be very interesting to test whether the NTC functions in the regulation of animal innate immunity.

\section{Materials and methods}

\section{Mutant screen and characterization of mos4}

All plants were grown under a $16 \mathrm{~h}$ light $/ 8 \mathrm{~h}$ dark regime. The mos suppressor screen and GUS staining protocol have been described previously (Zhang and Li 2005).

RNA used for gene expression analysis was extracted from 3-wk-old seedlings grown on Murashige and Skoog (MS) medium using the Totally RNA kit (Ambion). RT was carried out using the RT-for-PCR kit (Clontech). Real-time PCR was performed using the QuantiTect SYBR Green PCR kit (Qiagen). The primers used for amplification of Actin1, $P R-1$, and $P R-2$ were described previously (Zhang et al. 2003).

SA was extracted and measured from 4-wk-old soil-grown leaves using a previously described procedure (Li et al. 1999), proportionally scaled down using $100 \mathrm{mg}$ of tissue per sample. Four-week-old soil-grown Arabidopsis plants were infected with P.s.m. ES4326. Leaf discs of $0.32 \mathrm{~cm}^{2}$ were taken with a standard paper hole-punch. Infection of plants with H.p. Noco2 and H.p. Emwal was performed on 2-wk-old seedlings. These procedures were as described ( $\mathrm{Li}$ et al. 1999).

\section{Subcellular localization of MOS4}

The Gateway LR Clonase enzyme (Invitrogen) was used to catalyze the recombination between pENTR-gMOS4 (see the Supplemental Material) and a destination vector that is a modified binary vector with TOPO destination recombination sites with GFP fused in-frame to the C terminus. The MOS4-GFP expression clone was sequenced to confirm in-frame fusion and a lack of PCR errors. Transgenic plants were selected on MS medium containing $75 \mu \mathrm{g} / \mathrm{mL}$ kanamycin. Guard cells of transgenic seedlings were examined for GFP fluorescence as described previously (Y. Zhang et al. 2005).

\section{Yeast two-hybrid assay}

We used the GAL4-based Y2H system described previously (Kohalmi et al. 1998). Briefly, a GAL4(DB)-cMOS4 construct (see the Supplemental Material) was introduced into $\mathrm{Y} 2 \mathrm{H}$ strain YPB2 and used to screen an Arabidopsis cDNA expression library (kindly provided by William Crosby) encoding Arabidopsis proteins as C-terminal fusions to the GAL4 transcription activation domain (TA). Putative MOS4-interacting proteins were selected on the basis of histidine prototrophy and further screened on the basis of lacZ expression.

\section{In planta protein interaction assay}

Nuclear extracts (see the Supplemental Material) of transgenic mos4-1 complemented with gMOS4-GFP or gMOS4-HA and prl1-1 complemented with gPRL1-GFP or gPRL1-HA were mixed with $50 \mu \mathrm{L}$ of Anti-GFP or Anti-HA MicroBeads (Miltenyi Biotec) to magnetically absorb the epitope-tagged target protein. After an overnight incubation on ice, the MicroBead-bound target protein was magnetically precipitated on columns according to the manufacturer's instructions (uMACs; Miltenyi Biotec) for subsequent immunoblotting analysis. Immunoprecipitated proteins eluted from the column were detected with anti-GFP or anti-HA antibody, respectively, and the coimmunoprecipitated endogenous AtCDC5 protein was detected by a polyclonal antibody raised against the N-terminal 144-aminoacid fragment of AtCDC5.

\section{Acknowledgments}

We thank Gary Wong, Cristina Cotea, Larisa Aurelian, and Jack Zheng for their technical assistance; Dr. Sheng Yang He (Michigan State University) for the P.s.t. hrpA- mutant strain; Dr. Silke Robatzek (MPIZ, Cologne) for fls2 seeds and suggestions for flg22 experiments; and Dr. Jim Kronstad, Dr. Marcel Wiermer, and Dr. Sandra Goritschnig for critical reading of the manuscript. Financial support comes from the Natural Sciences and Engineering Research Council of Canada (NSERC), the Canadian Foundation for Innovation (CFI), British Columbia Knowledge Development Fund (BCKDF), UBC Blusson Fund, and Michael Smith Laboratories (UBC).

\section{References}

Aarts, N., Metz, M., Holub, E., Staskawicz, B.J., Daniels, M.J., and Parker, J.E. 1998. Different requirements for EDS1 and 
NDR1 by disease resistance genes define at least two R genemediated signaling pathways in Arabidopsis. Proc. Nat1. Acad. Sci. 95: 10306-10311.

Ajuh, P., Kuster, B., Panov, K., Zomerdijk, J.C., Mann, M., and Lamond, A.I. 2000. Functional analysis of the human CDC5L complex and identification of its components by mass spectrometry. EMBO J. 19: 6569-6581.

Ajuh, P., Sleeman, J., Chusainow, J., and Lamond, A.I. 2001. A direct interaction between the carboxyl-terminal region of CDC5L and the WD40 domain of PLRG1 is essential for pre-mRNA splicing. J. Biol. Chem. 276: 42370-42381.

Akira, S., Uematsu, S., and Takeuchi, O. 2006. Pathogen recognition and innate immunity. Cell 124: 783-801.

Austin, M.J., Muskett, P., Kahn, K., Feys, B.J., Jones, J.D., and Parker, J.E. 2002. Regulatory role of SGT1 in early R genemediated plant defenses. Science 295: 2077-2080.

Ausubel, F.M. 2005. Are innate immune signaling pathways in plants and animals conserved? Nat. Immunol. 6: 973-979.

Azevedo, C., Sadanandom, A., Kitagawa, K., Freialdenhoven, A., Shirasu, K., and Schulze-Lefert, P. 2002. The RAR1 interactor SGT1, an essential component of R gene-triggered disease resistance. Science 295: 2073-2076.

Bartsch, M., Gobbato, E., Bednarek, P., Debey, S., Schultze, J.L., Bautor, J., and Parker, J.E. 2006. Salicylic acid-independent ENHANCED DISEASE SUSCEPTIBILITY1 signaling in Arabidopsis immunity and cell death is regulated by the monooxygenase FMO1 and the Nudix hydrolase NUDT7. Plant Cell 18: 1038-1051.

Belkhadir, Y., Subramaniam, R., and Dangl, J.L. 2004. Plant disease resistance protein signaling: NBS-LRR proteins and their partners. Curr. Opin. Plant Biol. 7: 391-399.

Chisholm, S.T., Coaker, G., Day, B., and Staskawicz, B.J. 2006. Host-microbe interactions: Shaping the evolution of the plant immune response. Cell 124: 803-814.

Dinesh-Kumar, S.P. and Baker, B.J. 2000. Alternatively spliced $\mathrm{N}$ resistance gene transcripts: Their possible role in tobacco mosaic virus resistance. Proc. Nat1. Acad. Sci. 97: 19081913.

Dong, X. 2004. NPR1, all things considered. Curr. Opin. Plant Biol. 7: 547-552.

Eckmann, L. and Karin, M. 2005. NOD2 and Crohn's disease: Loss or gain of function? Immunity 22: 661-667.

Felix, G., Duran, J.D., Volko, S., and Boller, T. 1999. Plants have a sensitive perception system for the most conserved domain of bacterial flagellin. Plant J. 18: 265-276.

Gomez-Gomez, L., Felix, G., and Boller, T. 1999. A single locus determines sensitivity to bacterial flagellin in Arabidopsis thaliana. Plant J. 18: 277-284.

Grant, M.R., Godiard, L., Straube, E., Ashfield, T., Lewald, J., Sattler, A., Innes, R.W., and Dangl, J.L. 1995. Structure of the Arabidopsis RPM1 gene enabling dual specificity disease resistance. Science 269: 843-846.

Grillari, J., Ajuh, P., Stadler, G., Loscher, M., Voglauer, R., Ernst, W., Chusainow, J., Eisenhaber, F., Pokar, M., Fortschegger, $\mathrm{K}$., et al. 2005. SNEV is an evolutionarily conserved splicing factor whose oligomerization is necessary for spliceosome assembly. Nucleic Acids Res. 33: 6868-6883.

Hirayama, T. and Shinozaki, K. 1996. A $c d c 5^{+}$homolog of a higher plant, Arabidopsis thaliana. Proc. Natl. Acad. Sci. 93: 13371-13376

Hubert, D.A., Tornero, P., Belkhadir, Y., Krishna, P., Takahashi, A., Shirasu, K., and Dangl, J.L. 2003. Cytosolic HSP90 associates with and modulates the Arabidopsis RPM1 disease resistance protein. EMBO J. 22: 5679-5689.

Jordan, T., Schornack, S., and Lahaye, T. 2002. Alternative splicing of transcripts encoding Toll-like plant resistance pro-
teins-What's the functional relevance to innate immunity? Trends Plant Sci. 7: 392-398.

Katiyar-Agarwal, S., Morgan, R., Dahlbeck, D., Borsani, O., Villegas Jr., A., Zhu, J.K., Staskawicz, B.J., and Jin, H. 2006. A pathogen-inducible endogenous siRNA in plant immunity. Proc. Natl. Acad. Sci. 103: 18002-18007.

Kohalmi, S.E., Reader, L.J.W., Samach, A., Nowak, J., Haughn, G.W., and Crosby, W.L. 1998. Identification and characterization of protein interactions using the yeast 2-hybrid system. In Plant molecular biology manual M1 (eds. S.B. Gelvin and R.A. Schilperoort), pp. 1-30. Kluwer Academic Publishers, Dordrecht, The Netherlands.

Lee, S., Ha, S., Chung, M., Kim, Y., and Choi, Y. 2002. Mouse DAM1 regulates pro-apoptotic activity of BLK in mammary epithelial cells. Cancer Lett. 188: 121-126.

Li, X., Zhang, Y., Clarke, J.D., Li, Y., and Dong, X. 1999. Identification and cloning of a negative regulator of systemic acquired resistance, SNI1, through a screen for suppressors of npr1-1. Cell 98: 329-339.

Loscher, M., Fortschegger, K., Ritter, G., Wostry, M., Voglauer, R., Schmid, J.A., Watters, S., Rivett, A.J., Ajuh, P., Lamond, A.I., et al. 2005. Interaction of U-box E3 ligase SNEV with PSMB4, the $\beta 7$ subunit of the $20 \mathrm{~S}$ proteasome. Biochem. J. 388: 593-603.

Lu, M., Tang, X.Y., and Zhou, J.M. 2001. Arabidopsis NHO1 is required for general resistance against Pseudomonas bacteria. Plant Cell 13: 437-447.

Maass, N., Rosel, F., Schem, C., Hitomi, J., Jonat, W., and Nagasaki, K. 2002. Amplification of the BCAS2 gene at chromosome 1p13.3-21 in human primary breast cancer. Cancer Lett. 185: 219-223.

Mahajan, K.N. and Mitchell, B.S. 2003. Role of human Pso4 in mammalian DNA repair and association with terminal deoxynucleotidyl transferase. Proc. Natl. Acad. Sci. 100: 10746-10751.

Navarro, L., Dunoyer, P., Jay, F., Arnold, B., Dharmasiri, N., Estelle, M., Voinnet, O., and Jones, J.D. 2006. A plant miRNA contributes to antibacterial resistance by repressing auxin signaling. Science 312: 436-439.

Nemeth, K., Salchert, K., Putnoky, P., Bhalerao, R., Koncz-Kalman, Z., Stankovic-Stangeland, B., Bako, L., Mathur, J., Okresz, L., Stabel, S., et al. 1998. Pleiotropic control of glucose and hormone responses by PRL1, a nuclear WD protein, in Arabidopsis. Genes \& Dev. 12: 3059-3073.

Neubauer, G., King, A., Rappsilber, J., Calvio, C., Watson, M., Ajuh, P., Sleeman, J., Lamond, A., and Mann, M. 1998. Mass spectrometry and EST-database searching allows characterization of the multi-protein spliceosome complex. Nat. Genet. 20: 46-50.

Ohi, M.D. and Gould, K.L. 2002. Characterization of interactions among the Cef1p-Prp19p-associated splicing complex. RNA 8: 798-815.

Philpott, D.J. and Girardin, S.E. 2004. The role of Toll-like receptors and Nod proteins in bacterial infection. Mol. Immunol. 41: 1099-1108.

Qi, C., Zhu, Y.T., Chang, J., Yeldandi, A.V., Rao, M.S., and Zhu, Y.J. 2005. Potentiation of estrogen receptor transcriptional activity by breast cancer amplified sequence 2. Biochem. Biophys. Res. Commun. 328: 393-398.

Savaldi-Goldstein, S., Aviv, D., Davydov, O., and Fluhr, R. 2003. Alternative splicing modulation by a LAMMER kinase impinges on developmental and transcriptome expression. Plant Cell 15: 926-938.

Swiezewski, S., Crevillen, P., Liu, F., Ecker, J.R., Jerzmanowski, A., and Dean, C. 2007. Small RNA-mediated chromatin silencing directed to the $3^{\prime}$ region of the Arabidopsis gene 
encoding the developmental regulator, FLC. Proc. Natl. Acad. Sci. 104: 3633-3638.

Tani, A. and Murata, M. 2005. Alternative splicing of Pot1 (Protection of telomere)-like genes in Arabidopsis thaliana. Genes Genet. Syst. 80: 41-48.

Tarn, W.Y., Lee, K.R., and Cheng, S.C. 1993. The yeast PRP19 protein is not tightly associated with small nuclear RNAs, but appears to associate with the spliceosome after binding of U2 to the pre-mRNA and prior to formation of the functional spliceosome. Mol. Cell. Biol. 13: 1883-1891.

Tsai, W.Y., Chow, Y.T., Chen, H.R., Huang, K.T., Hong, R.I., Jan, S.P., Kuo, N.Y., Tsao, T.Y., Chen, C.H., and Cheng, S.C. 1999. Ceflp is a component of the Prp19p-associated complex and essential for pre-mRNA splicing. J. Biol. Chem. 274: 9455-9462.

Tzafrir, I., Dickerman, A., Brazhnik, O., Nguyen, Q., McElver, J., Frye, C., Patton, D., and Meinke, D. 2003. The Arabidopsis SeedGenes Project. Nucleic Acids Res. 31: 90-93.

van der Biezen, E.A., Freddie, C.T., Kahn, K., Parker, J.E., and Jones, J.D. 2002. Arabidopsis RPP4 is a member of the RPP5 multigene family of TIR-NB-LRR genes and confers downy mildew resistance through multiple signalling components. Plant J. 29: 439-451.

Zhang, X.C. and Gassmann, W. 2003. RPS4-mediated disease resistance requires the combined presence of RPS4 transcripts with full-length and truncated open reading frames. Plant Cell 15: 2333-2342.

Zhang, Y. and Li, X. 2005. MOS3, encoding a putative Nucleoporin 96, is required for both basal defense and constitutive resistance responses mediated by snc1. Plant Cell 17: 13061316.

Zhang, Y., Goritschnig, S., Dong, X., and Li, X. 2003. A gain-offunction mutation in a plant disease resistance gene leads to constitutive activation of downstream signal transduction pathways in suppressor of npr1-1, constitutive 1. Plant Cell 15: 2636-2646.

Zhang, N., Kaur, R., Lu, X., Shen, X., Li, L., and Legerski, R.J. 2005. The Pso4 mRNA splicing and DNA repair complex interacts with WRN for processing of DNA interstrand cross-links. J. Biol. Chem. 280: 40559-40567.

Zhang, Y., Cheng, Y.T., Bi, D., Palma, K., and Li, X. 2005. MOS2, a protein containing G-patch and KOW motifs, is essential for innate immunity in Arabidopsis thaliana. Curr. Biol. 15: 1936-1942.

Zhou, Z., Licklider, L.J., Gygi, S.P., and Reed, R. 2002. Comprehensive proteomic analysis of the human spliceosome. $\mathrm{Na}$ ture 419: 182-185. 


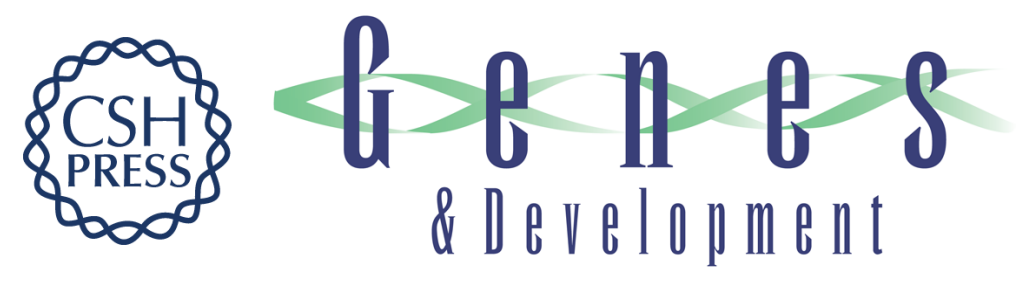

\section{Regulation of plant innate immunity by three proteins in a complex conserved across the plant and animal kingdoms}

Kristoffer Palma, Qingguo Zhao, Yu Ti Cheng, et al.

Genes Dev. 2007, 21:

Access the most recent version at doi:10.1101/gad.1559607

Supplemental
Material http://genesdev.cshlp.org/content/suppl/2007/06/12/21.12.1484.DC1

References This article cites 46 articles, 24 of which can be accessed free at:

http://genesdev.cshlp.org/content/21/12/1484.full.html\#ref-list-1

License

Email Alerting Receive free email alerts when new articles cite this article - sign up in the box at the top

Service

right corner of the article or click here.

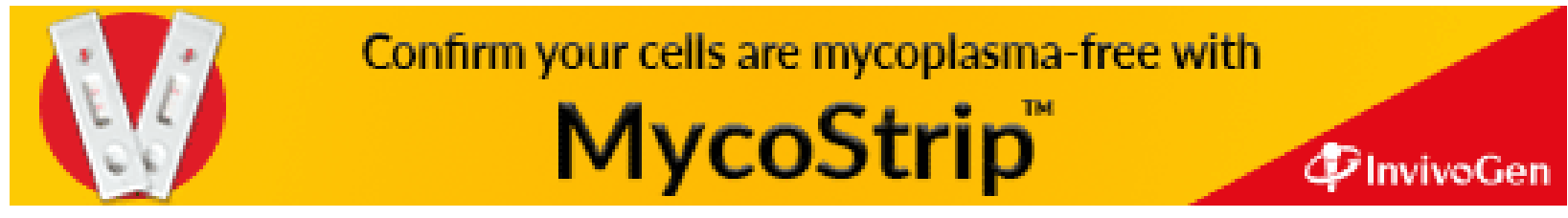

